\section{EMBRYAIDDLE Aeronautical University}

SCHOLARLY COMMONS
International Journal of Aviation, Aeronautics, and Aerospace

\title{
Internet of Things 36-rotor Multicopter for lonizing Radiation Surveying
}

\author{
Svetoslav Zabunov \\ Space Research and Technology Institute -- Bulgarian Academy of Sciences, \\ svetoslavzabunov@gmail.com \\ Garo Mardirossian \\ Space Research and Technology Institute -- Bulgarian Academy of Sciences, \\ garo.mardirossian@gmail.com \\ Rositsa Miteva \\ Institute of Astronomy and National Astronomical Observatory -- Bulgarian Academy of Sciences, \\ rmiteva@nao-rozhen.org \\ Todor Kunchev \\ Faculty of Medicine - Sofia University, drtodorkunchev@yahoo.co.uk
}

Follow this and additional works at: https://commons.erau.edu/ijaaa

Part of the Aeronautical Vehicles Commons, Nuclear Engineering Commons, and the Systems Engineering and Multidisciplinary Design Optimization Commons

\section{Scholarly Commons Citation}

Zabunov, S., Mardirossian, G., Miteva, R., \& Kunchev, T. (2021). Internet of Things 36-rotor Multicopter for Ionizing Radiation Surveying. International Journal of Aviation, Aeronautics, and Aerospace, 8(2). https://doi.org/10.15394/ijaaa.2021.1585

This Concept Paper is brought to you for free and open access by the Journals at Scholarly Commons. It has been accepted for inclusion in International Journal of Aviation, Aeronautics, and Aerospace by an authorized administrator of Scholarly Commons. For more information, please contact commons@erau.edu. 


\section{Internet of Things 36-rotor Multicopter for Ionizing Radiation Surveying}

\section{Cover Page Footnote}

We would like to thank Prof. Jordanka Semkova from the Space Research and Technology Institute Bulgarian Academy of Sciences for giving us valuable advice during the course of this thrilling research. 
After the initial rapid development of multicopters during the last decade (Battsengel et al., 2020; Kovalev et al., 2019), a tendency towards implementing drones in specific environments is on the rise (Nex \& Remondino, 2019). For indoor task solving, smaller by size and weight unmanned aerial vehicles (UAVs) are required (Croon \& Wagter, 2018; Khosiawan \& Nielsen, 2016; Molina et al., 2018). Another trend in UAV innovations is increasing the number of rotors with the purpose of attaining certain benefits thereof (Zabunov \& Mardirossian, 2018).

The herein presented drone design is suitable for large and small drones and is applicable to various tasks and carrying different kinds of payload, but we see the major purpose of implementation of this aircraft in ionization radiation sources surveying within buildings. Hence, we have directed our efforts towards making the smallest possible multirotor that is still capable of carrying various radiological sensors as payloads.

Our 36-rotor UAV is built around a few novel ideas that improve its characteristics both when involved in general tasks and when fulfilling its main purpose - radiological surveying. These novel ideas are elaborated on in the next section. The used radiological instrument onboard the UAV is also developed by the authors and is described further on in the present article.

The application is in radiation related disasters such as the Fukushima Daiichi nuclear disaster of 2011 (Kawatsuma et al., 2012) or the Chernobyl disaster of 1986 (Berger, 2010). In such scenarios there is a need for containing the dissemination of radioactive materials inside buildings and the surrounding terrain. Another task is to investigate the contamination of natural resources in physical proximity to the incident. For the solution of these problems, one could employ robotic platforms. They are used to search, survey, and map the dissemination of the radioactive materials and estimate the radiation dose at different locations inside the affected region.

Another application may be the detection and control by law enforcement officials and customs personnel of illegally transported and stored radioactive materials. 


\section{Figure 1}

The 36-rotor Multicopter Prototype Design

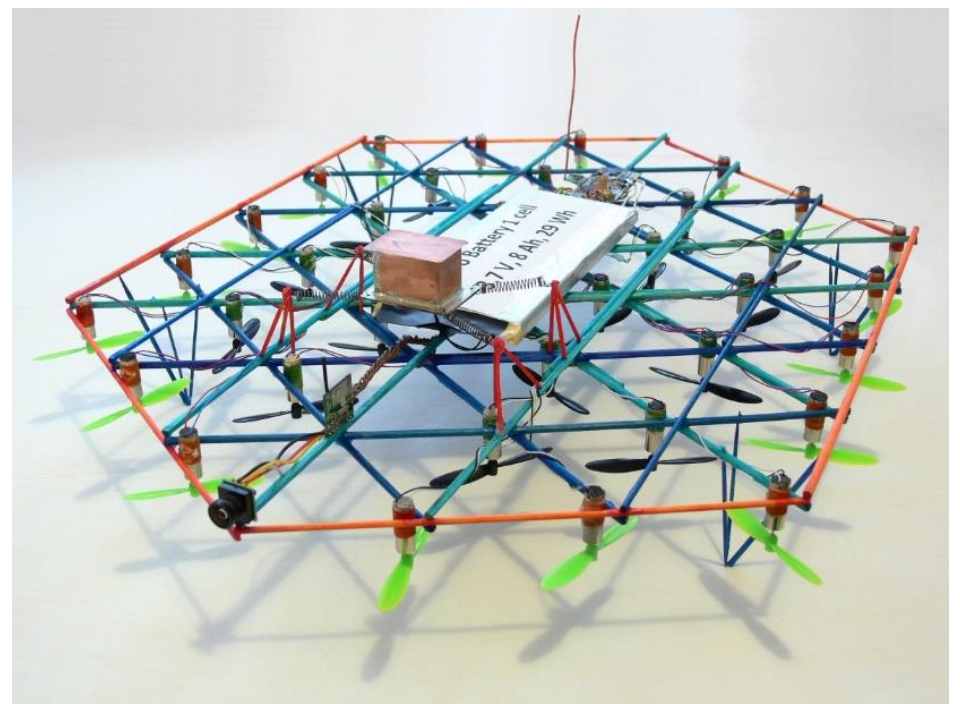

Note. Total weight of the craft is $450 \mathrm{~g}$. Dimensions: 455 x 430 x $100 \mathrm{~mm}$.

\section{Related Existing Projects}

There are existing robotic platforms developed with the purpose of radiological surveying. These fall into mainly two categories: ground based and airborne systems. For very specific situations, sailing robotized vessels may be engaged. For use inside buildings only ground based robots have been developed so far with few exceptions. Examples of ground-based robots for radiological surveying are the CARMA 2 platform presented by Bird et al. (2019), the JAEA-3 robot described by Kawatsuma et al. (2017), and the Quince robot by Nagatani et al. (2013). The development of these robots was motivated by the Fukushima Daiichi nuclear disaster. Another ground-based robot is the proposed design by Chaiyasoonthom et al. (2015). It is a tracked robot equipped with Geiger Müller tube for $\gamma$-and $\beta$-surveying. Zakaria et al. (2016) have developed a three wheeled robotic platform utilizing again a Geiger Müller counter. One more tracked robot was proposed by Kim et al. (2017). Still another three wheeled robot was presented by Dudar et al. (1994) in a U.S. patent. We should also mention the somewhat successful use of Lunokhod during the Chernobyl disaster (Zarowny, 2011). The robots had radiation hardened electronics and were protected from the radiation levels met on the Moon surface. Nevertheless, due to the extreme radiation levels at the Chernobyl site, the employed two machines failed some period of time after their engagement (Anderson, 1990).

A good overview of the existing airborne radiation mapping systems is carried out by Connor et al. (2016). The drones in their research have total weights 
above $1 \mathrm{~kg}$ and are not suitable for indoor flying except one design - the solution proposed by Boudergui et al. (2011) consists of a $0.9 \mathrm{~kg}$ indoor flying UAV for radiological observations carrying two different sensors weighing $20 \mathrm{~g}$ and $70 \mathrm{~g}$ respectively. The drone is a standard design - a quadcopter using brushless electric motors and a Li-Po battery. Martin et al. (2016) elaborated on a standard design for outdoors flying and mapping of the environment for radiological sources. As such, the project is developed around a multicopter carrying a $200 \mathrm{~g}$ radiological sensor. The UAV has total weight of $7 \mathrm{~kg}$ and is navigated by a GPS system. The procedure of mapping is carried out by flying along a predefined route.

A fixed wing solution that covers large areas and works well in windy and harsh conditions was proposed by Connor et al. (2020). Still another platform for outdoor flying was proposed by Mochizuki et al. (2017). Their development is intended for use in the Fukushima-Daiichi accident site. They are employing a commercial drone with total weight of $4.2 \mathrm{~kg}$ and $1 \mathrm{~m}$ maximum dimensions. The mounted payload is a compact Compton camera weighing only $1.9 \mathrm{~kg}$.

After investigating the existing flying platforms for radiological surveying, we were not able to identify many indoor drones for this purpose (Boudergui et al., 2011). The solutions are mostly based on commercial drones and existing radiological instruments that were mounted to the drones as payload. This approach offers quick results but responding to stringent requirements for surveying radiation sources inside buildings requires a design purposely developed for that task. 
Figure 2

Airframe Design of the 36-Rotor Drone

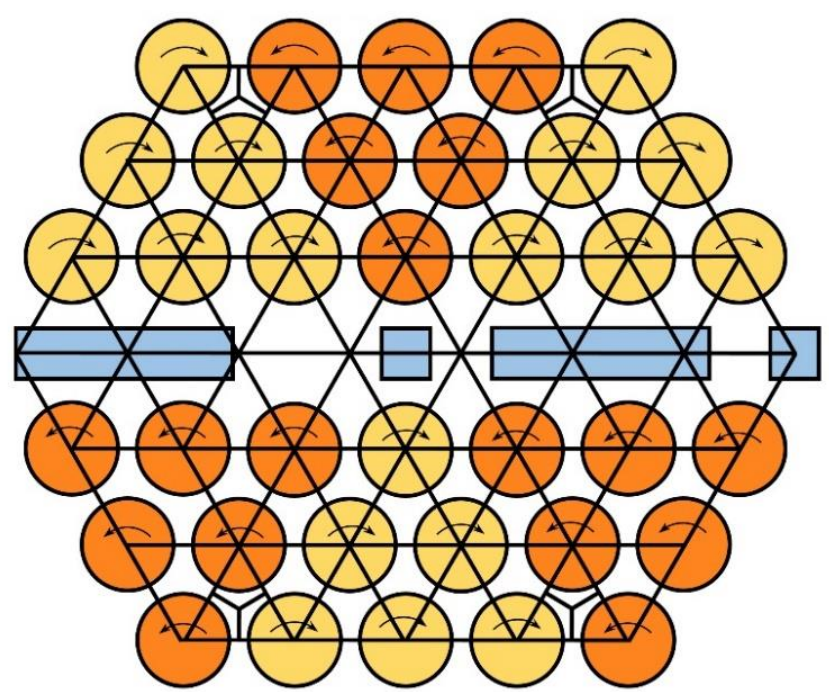

Note. The rotors are positioned using a honeycomb geometric covering. With yellow colour are marked the clockwise turning rotors, with orange colour - the counter-clockwise rotating ones. The blue boxes show other avionics modules and payload compartments.

Most of the existing robots for radiological surveying are developed around non radiation hardened/resistant electronics. Nevertheless, these systems have high costs (Bird et al., 2019). It is true that cost is relative and depends on the situation, but we should note that having electronics vulnerable to radiation will presume a high frequency failure rate. This drawback combined with a high unit cost renders such a project extremely expensive to operate.

As it was already mentioned, the large weight of most existing flying platforms prohibits their use inside buildings. The few designed for that purpose are still heavy $(0.9 \mathrm{~kg})$ and lower total weight may be chased for. Considering the pointed-out shortcomings of the present technology we suggest a novel airborne platform addressing these problems.

\section{The 36-Rotor Multicopter Design and Prototype Development}

The design is an electrically powered multirotor aircraft employing brushed motors and constant pitch propellers. We have chosen to utilize as large a number of rotors in our UAV as feasible. Thirty-six (36) was the number of rotors advised by the geometric symmetry in accord with the chosen spatial rotor distribution as explained below. Thirty-six rotors are also divisible by 6 as our aim was to use 6 channels to control the rotors. An even larger number of rotors is possible, but in order to keep the dimensions of the aircraft within reasonable limits we estimated 
that we cannot go any higher. If, still, a larger number of rotors is to be used, the motors should be chosen smaller in size. This would render the design extremely complex to prototype, test, and maintain.

Figure 3

Airframe of the Prototype

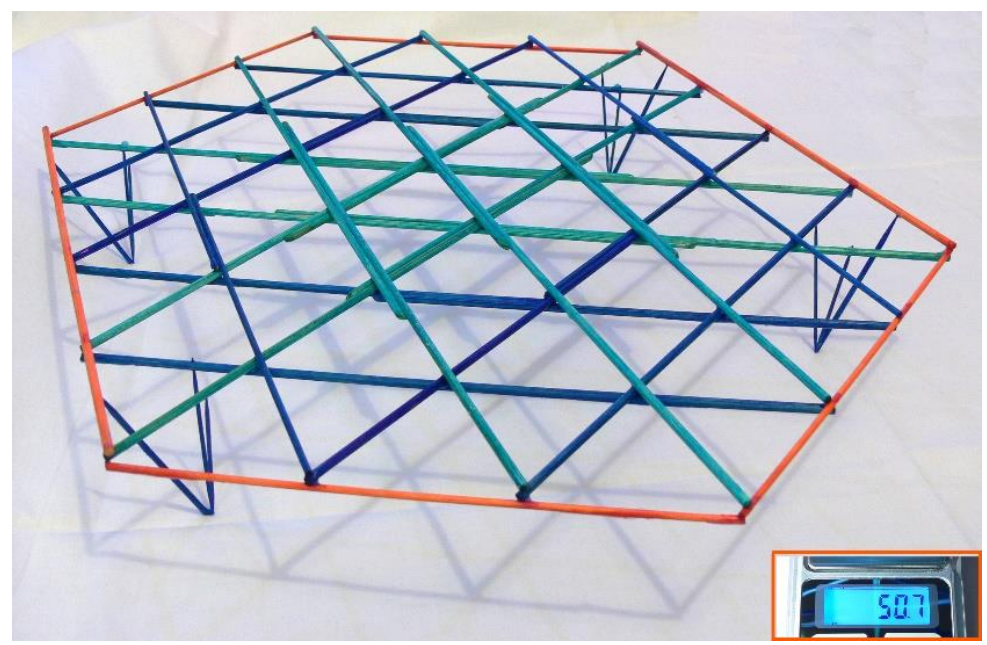

Note. Weight of the airframe is $50.7 \mathrm{~g}$.

In comparison to standard designs, we see in everyday usage such as 4,6 , and 8 rotor drones, a larger number of rotors, in this case 36, results in rotor vibrations frequency bandwidth being shifted towards higher frequencies. This helps filter the mechanical vibrations better and using cheaper dumping constructions. Also, the audible noise the aircraft emits, when at higher frequencies, decays quicker with distance, making the UAV harder to spot by acoustic signature as noted in (Marichal et al., 2014; Radkowski \& Szulim, 2013; Verbeke \& Debruyne, 2016) and Piercy and Embleton (1977). Further benefits of the larger number of rotors include the lower risk of catastrophic failure should a single rotor malfunction. The final, but not least important benefit of using larger number of rotors, is that each rotor accumulates a smaller fraction of all rotors rotating kinetic energy. In such a way, if a given rotor disintegrates in flight the released kinetic energy through debris will be a smaller fraction of the total energy of all rotating parts in the UAV. The same logic holds if a rotor gets into contact with an object only the kinetic energy of that single rotor will interact with the object.

In our design, the 36 rotors are divided into 6 groups, each group consisting of 6 rotors turning in the same direction (see Figures 1 and 2). The control of the electric motors is realized using 6 channels control circuitry - one channel manages 6 rotors. Each 6 rotors in one channel are driven in parallel. Another novelty in our 
design is the airframe that is built according to the honeycomb optimal geometric coverage design (see Figures 2 and 3).

\section{Figure 4}

The Prototype Having the Motors and Propellers Mounted to the Airframe

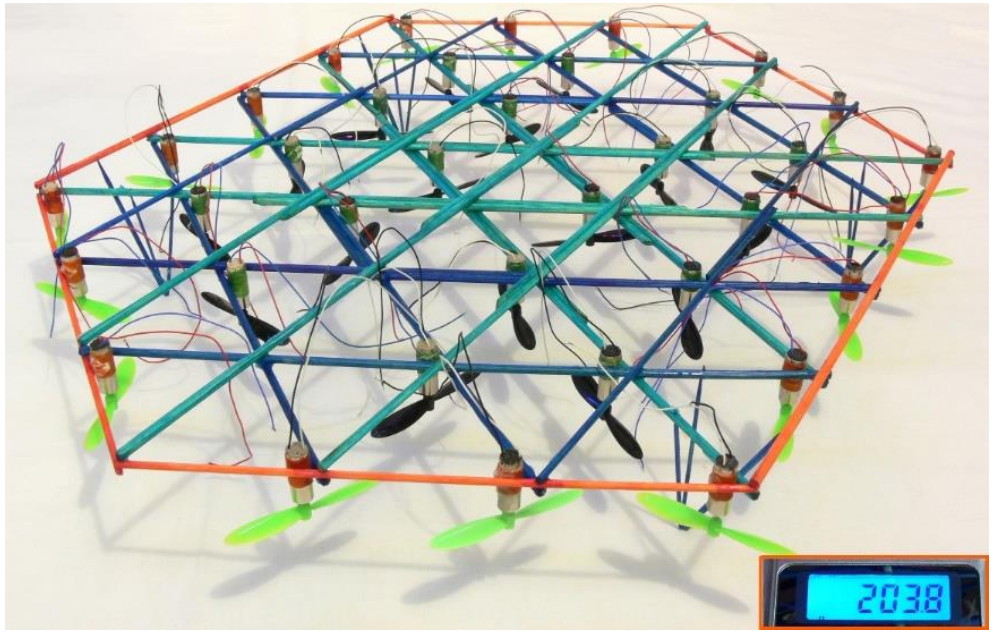

Note. The weight at this stage is $203.8 \mathrm{~g}$.

This design helps achieve maximum density of the rotors without overlapping of their rotor disks. Optimal geometric coverage is proven by the single plane densest circles packing also known as hexagonal honeycomb packing (Steinhaus, 1999). It has a packing density of:

$$
\eta_{h}=\frac{1}{6} \pi \sqrt{3} \approx 0.9069
$$

The honeycomb approach of rotor geometrical distribution was successfully implemented in a 12-rotor multicopter model (Zabunov \& Mardirossian, 2018). The so achieved compactness of the design helps lower the weight of the airframe and hence the total weight of the aircraft. Further, the dimensions of the aircraft are thus minimized - a property of immense value when navigating the drone inside buildings and passing through doors and windows.

The next novelty in our design is the mounting position of the rotors. They are mounted under the fuselage. For this purpose, we make use of pusher propellers. The undermount propeller approach was implemented by Yoon et al. (2017), Theys et al. (2016), and Zabunov and Mardirossian (2018). The electric motors in our design are attached to the fuselage in an undermount style and off-body (see Figure 1) with distance between the propellers and the motor hubs of 0.4 rotor radii. As previously tested while developing the 12-rotor aircraft (Zabunov \& Mardirossian, 
2018 ), if the propeller discs were positioned too close ( 0.2 rotor radii or less) to the airframe a significant propeller induced flow interference with the fuselage would occur and a degradation of the aerodynamic efficiency of lift would result (Theys et al., 2016). It was already mentioned that increasing the number of rotors in a multirotor aircraft favours the mechanical filtering of vibrations caused by the rotating propellers. When using undermount rotors the propeller induced vibrations in the airframe and related acoustic noise are further significantly diminished which translates to better aircraft stability and less interference with the payload (Yoon et al., 2017). In the case of undermount propellers, the lower vibrations and acoustic noise is due to the lesser propeller induced flow interference with the airframe. In contrast, in the case of overmount propellers, the propeller wash would lead to pulsating dynamic pressure on the fuselage and consequently created audible sound waves. The advantages of employing undermount pusher propellers were tested in an experimental 12-rotor aircraft proving a 7\% increase in lift efficiency with the tested 12-rotor design (Zabunov \& Mardirossian, 2018).

Our prototype development was not only based on the aforementioned ideas, but also on the task of indoor radiological surveying. The payload the drone should be able to carry was consisting of a radiological sensor. To be able to fly inside buildings and negotiate doors and windows, our prototype had to have certain maximum dimensions. After considering the dimensions of standard doors and windows we designed a prototype with dimensions of 455 x 430 x $100 \mathrm{~mm}$ (Figure 1). This limitation established the type of motors we selected to use - brushed micro motors with $7 \mathrm{~mm}$ diameter, $20 \mathrm{~mm}$ height and $0.9 \mathrm{~mm}$ shaft (see Figures 1 and 4). The employed propellers are $55 \mathrm{~mm}$ in diameter.

Another requirement for the prototype was to be low cost. A radiological surveillance robot is subjected to high radiation doses and harsh conditions, as noted above, and has high probability of malfunctioning. Lower cost of the UAV would enable the operator to employ a larger number of units in a given mission and treat them as disposable tools. 


\section{Figure 5}

\section{Avionics and Electrical Block Schematic}

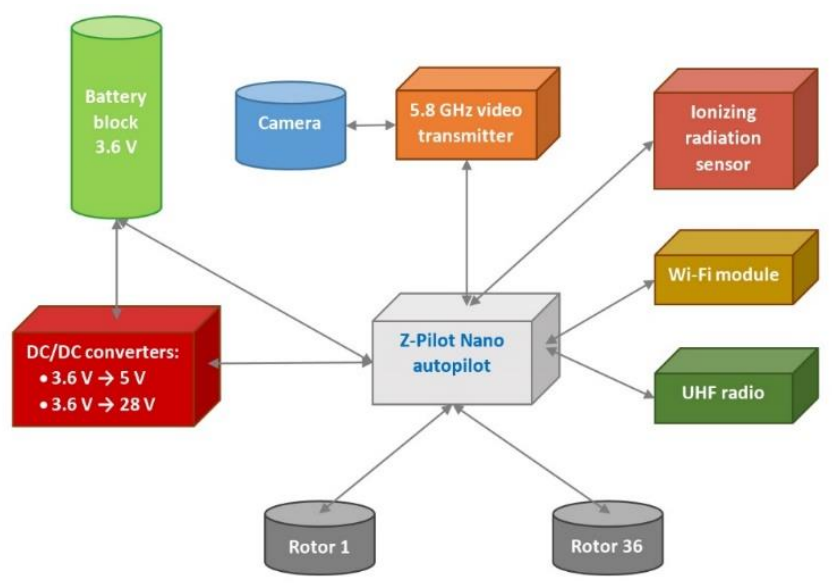

The prototype development started with the construction of the airframe (Figure 3). Its weight is $50.7 \mathrm{~g}$. The next step was to mount the motors and propellers (see Figure 4). With these fit the weight raised to $203.8 \mathrm{~g}$. By installing the rest of the avionics and the payload and battery, the weight totalled at $450 \mathrm{~g}$ (Figure 1).

Figure 5 shows the general diagram of the aircraft systems and avionics. In the centre of the electronics is the autopilot Z-Pilot Nano, developed by the first author Zabunov $(2016,2019)$. This autopilot weighs $5 \mathrm{~g}$ and its current version is capable of driving brushed motors in 6 separate channels.

An image of the autopilot onboard the 36-rotor aircraft prototype is shown in Figure 6 and its block diagram is depicted in Figure 7. The autopilot is developed around the ARM core NXP microcontroller model MK22FN1M0VLL12. This microcontroller has 32 bits architecture and exhibits digital signal processing capabilities such as a floating-point unit. It is manufactured in a 100-pin low-profile quad flat package (LQFP) with $0.5 \mathrm{~mm}$ pin pitch. The microcontroller offers two analogue to digital converters (ADCs) of the type successive approximation register (SAR). The maximum resolution of the ADCs is 16 bit. The processor offers 128 kiB random access memory (RAM) and $1 \mathrm{MiB}$ flash read only memory (ROM). The maximum operating frequency is $120 \mathrm{MHz}$ - a speed totally adequate for the purpose of autopilot design capable of performing additional tasks in parallel. 
Figure 6

Z-Pilot Nano

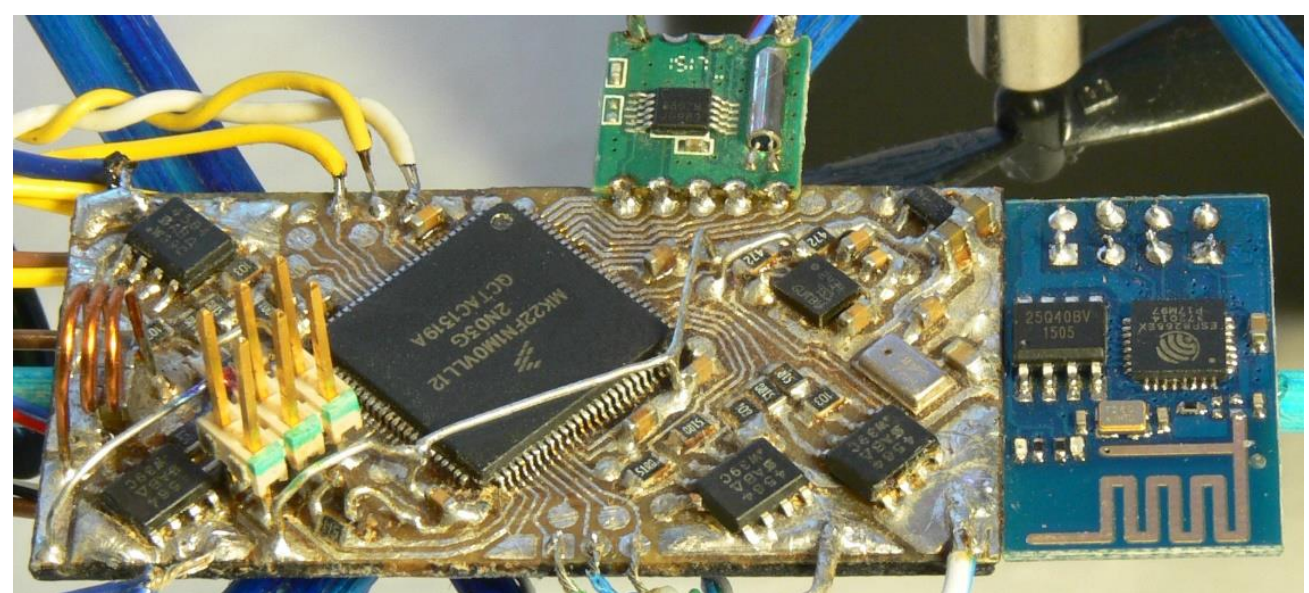

Note. Z-Pilot Nano autopilot along with the UHF radio receiver (top) and Wi-Fi module (right).

The processor is clocked using a crystal oscillator and stable frequency generation is at hand for the communication with other devices of the avionics that require it.

Figure 7

Autopilot Z-Pilot Nano Block Diagram

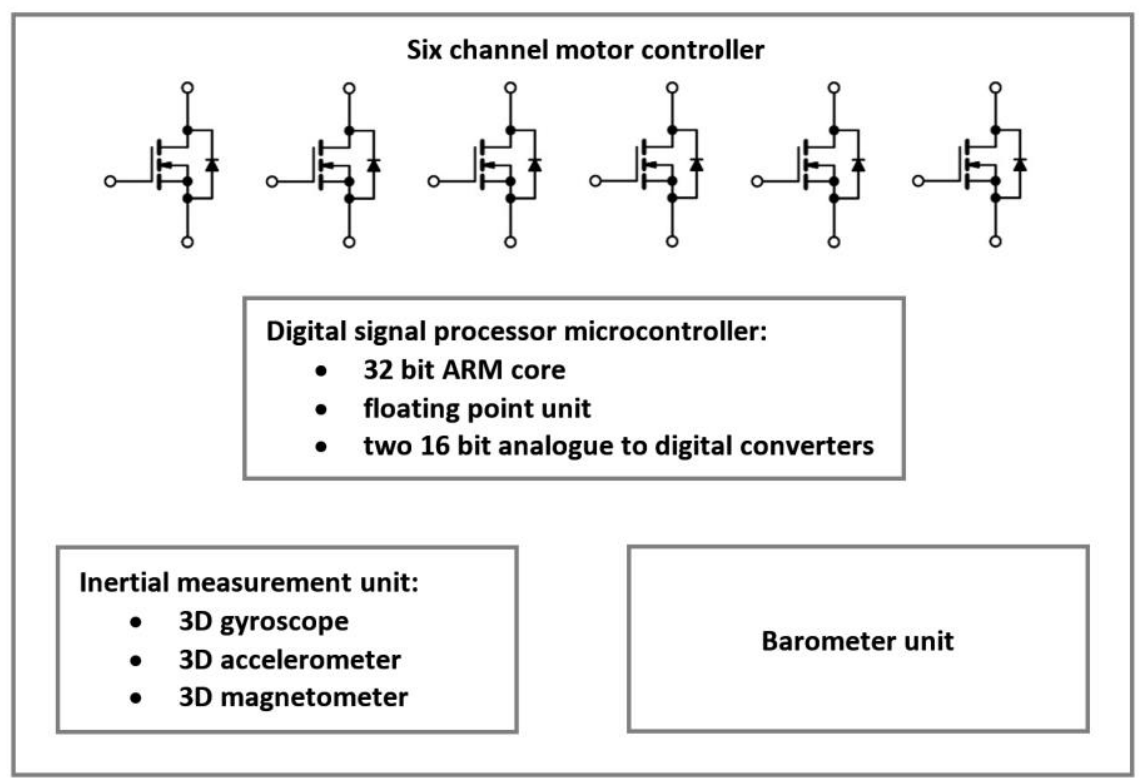


The motor control channels are also part of the autopilot and are realized using 6 metal oxide semiconductor field effect transistors (MOSFETs). The brushed electric motors are powered using pulse width modulation (PWM). By the help of PWM approach the autopilot is capable of controlling each channel's average voltage and the power output of the motors connected to that channel respectively.

The autopilot employs an inertial measurement unit (IMU) realized using microelectromechanical 3D gyroscope, 3D accelerometer and 3D magnetometer. A barometer is installed in the autopilot having altimeter resolution of $10 \mathrm{~cm}$.

The rest of the electronics include the drone power source - a Li-Ion or LiPo battery with nominal voltage of $3.6 \mathrm{~V}$ and $3.7 \mathrm{~V}$ respectively. Our prototype uses a Li-Po battery with capacity of $8 \mathrm{Ah}$, but Li-Ion batteries of the 18650 standard are applicable and better suited for their higher energy density. For the purpose of powering different schematics onboard, voltage step-up converters are installed. $5 \mathrm{~V}$ is needed for the video transmitter, while the radiological sensor requires additional $28 \mathrm{~V}$ supply.

\section{Figure 8}

Radio Communications on Board of the Multirotor

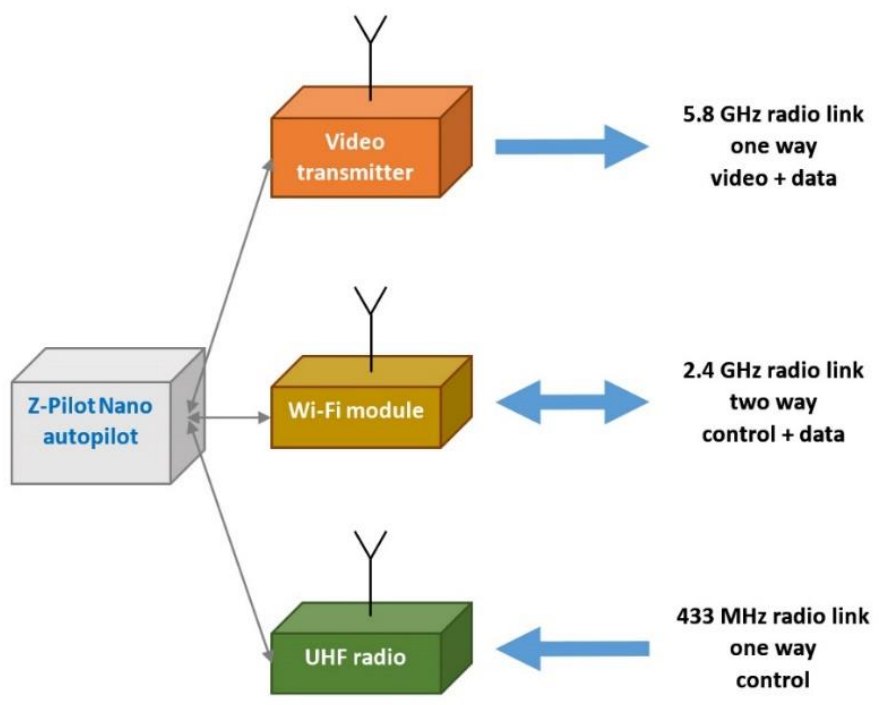

The camera installed is a standard NTSC video camera. It is connected to a $5.8 \mathrm{GHz}$ video transmitter whose stereo audio channels are used for data transmission from the aircraft (a one-way radio link). Another one-way radio link but used to transfer control signals to the aircraft is realized using a small ultra-high frequency (UHF) receiver module (see Figure 6 - top). The third radio link is a twoway radio module employed in the realization of a Wi-Fi connection (see Figure 6 - 
right). The Wi-Fi radio connection is redundant and may be employed in place of the other radio links or simultaneously with them in order to diversify the communication data transfer and improve the bandwidth and reliability of the information exchange with the drone. The Wi-Fi radio link effectively enables the drone to work as an Internet of things (IoT) device. For a block diagram summary of the used radio links see Figure 8.

The last part of the electronics is the ionizing radiation sensor. It is scrutinized in more detail in the next section.

\section{Ionizing Radiation Sensor}

For the purpose of ionizing radiation surveying by means of robotized platforms we have developed a radiological sensor suitable for small sized robots. The main motivation was to enable robots penetrate buildings and perform investigation indoors. Further condition was the sensor to be applicable to ground based and flying platforms which raised the requirements for the device's weight. Hence we accepted an upper limit of $10 \mathrm{~g}$ weight for the sensor. In order to achieve this goal an analysis of the available ionizing sensor technologies was carried out (Garcia-Sanchez et al., 2018).

Figure 9

Radiological Sensor Diagram

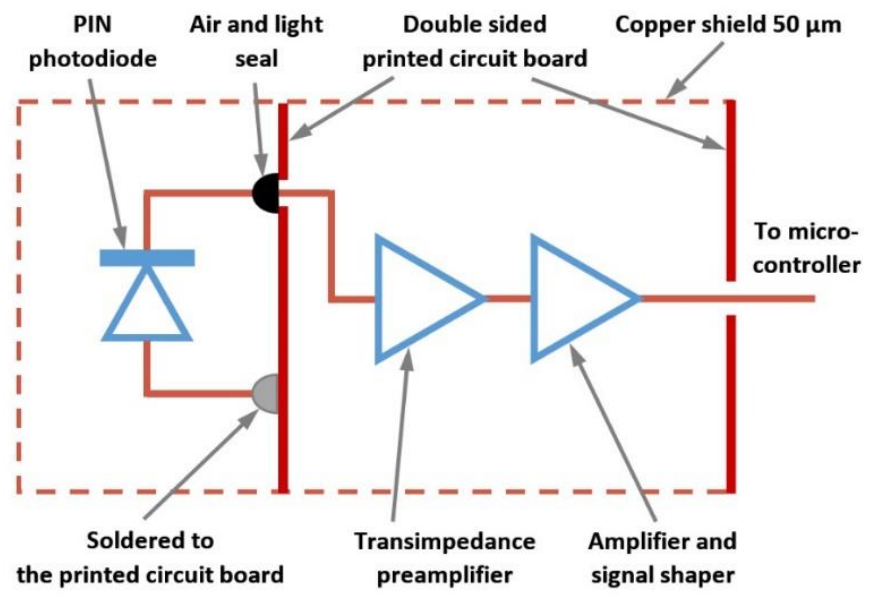

The expected sensor ability to register ionizing particles was defined as minimum and maximum requirements. The minimum requirement was defined as registering gamma and beta particles while the maximum one was to sense gamma and beta particles, neutrons, accelerated protons, and accelerated heavier ions. Although radiation sources not emitting gamma and beta particles are rare, an ability of the device to register broader variety of particles is an advantage. Alpha 
particles are not in our interest at this moment, because their penetration in air is quite limited and searching for radiation sources by alpha emission will not be fruitful. Nevertheless, a future variant of the instrument might include an alpha sensing module for the purpose of radiation source identification and danger estimation.

Another function that was to be offered by the instrument was a spectrometer function. It is used to identify the type of the radiation source. The last requirement was to keep the cost low and it was established along the same low-cost requirement as for the whole flying machine.

By studying the technologies applicable to electronic radiological surveying, we identified two groups of instruments: gas detectors and solid-state detectors. The first technology precedes chronologically the solid-state devices and involves the usage of gas filled tubes inside of which high voltage potential is established. Solid state detectors, on the other hand, are using semiconductor devices, in most cases PIN photodiodes, and scintillator materials. What follows is an analysis of the available technologies (Garcia-Sanchez et al., 2018) and their advantages and shortcomings in respect to our design requirements.

Figure 10

Ionizing Radiation Sensor

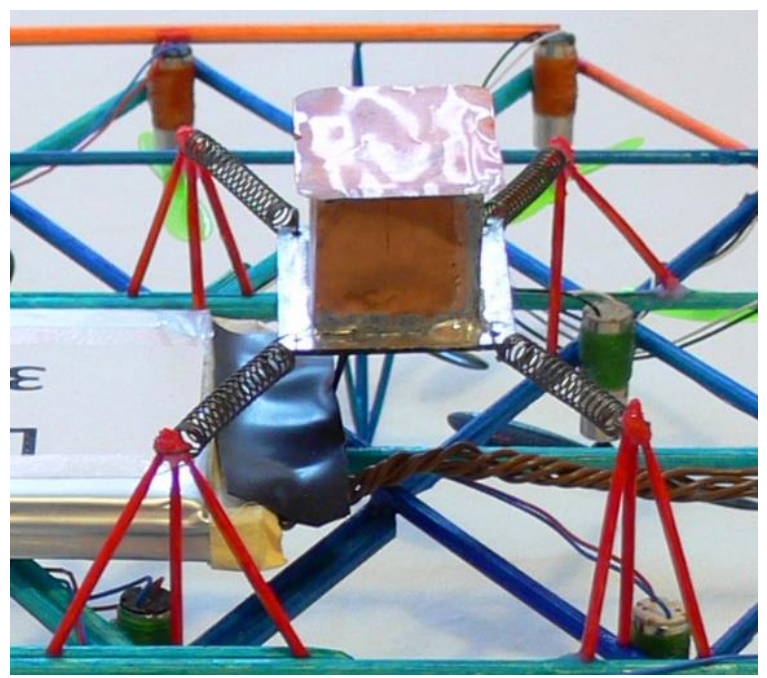

Note. Ionizing radiation sensor is mounted on the 36-rotor drone airframe using sprung suspension.

Geiger-Müller tubes are a type of gas detector. They are tubes filled with a special gas in the volume of which high voltage potential is established using two electrodes. When an ionizing radiation particle strikes the gas molecules the latter ionize. The ionized molecules and the knocked out electrons accelerate in the 
direction of the oppositely charged electrodes respectively. Due to the high acceleration, they ionize in an avalanche process other gas molecules on their way. An impulse of electric current occurs between the electrodes. Because of the avalanche process the current amplitude is not related to the ionizing particle energy and the Geiger-Müller tube cannot be used for spectrometry. It only counts the ionizing particles. Hence, this technology was rejected.

Another gas tube-based device is the ionization chamber. It again uses two electrodes with high voltage across. In most scenarios the used gas is air and the tube is open so that air could freely circulate inside it. Just like the Geiger-Müller tube, when a gas molecule is struck by an ionizing particle the molecule ionizes. Here the electric field is not as strong as with the Geiger-Müller apparatus. This prevents an avalanche effect (Shrivastava \& Henry, 2009). The ionization inside the chamber causes current to flow between the electrodes. The current is proportional to the intensity of the ionizing radiation. Neither counting nor spectral analysis is possible with this device; that is why it is unsuitable for our needs.

A third gas tube technology is the proportional counter. It is similar to the Geiger-Müller tube with the distinction that the voltage is so chosen that the impulse caused by the avalanche process has an amplitude proportional to the energy of the ionizing particle (Seco et al., 2009). This device is applicable as a spectrometer and a counter. We abandoned it because it is cumbersome, heavy, and expensive and involves very high voltages making the circuitry complex and harder to maintain.

From the solid-state detectors, we have examined the PIN photodiode and the scintillator-based devices. The PIN photodiode detector works by having the ionizing particles cause ionization in the semiconductor material resulting in current impulses to occur related to each ionization event. The impulses have amplitude proportional to the energy of the ionizing particle. This makes the PIN photodiode suitable for spectrometry and counting (Semkova et al., 2018). PIN photodiode-based detectors are capable of registering not only gamma and beta particles, but also accelerated protons and heavy ions, and neutrons with energies above $1 \mathrm{MeV}$ (Spur \& Dachev, 2003; Spurný, 2005; Spurný et al., 2009).

Scintillation detectors consist of transparent materials that scintillate upon ionizing particle bombardment. The light emitted in such an event is registered using a photo detector. For photo detectors traditionally photo multiplier tubes were employed. Recently with the advent of new scintillation materials photo diodes have become advantageous for this purpose. The intensity of the emitted light is proportional to the ionizing energy of the particle released inside the scintillation material. This property along with their very high efficiency make scintillators the best technology for spectrometry (Seco et al., 2009). We have identified as the best candidate for future development to be the caesium iodide doped with thallium scintillator. It is not only registering neutrons along with gamma and beta particles, 
but using signal shape analysis, separation between neutrons and gamma rays may be achieved (Ashida et al., 2018).

Overall, we identified two suitable technologies for our purposes - the PIN photodiode detector and the scintillator-based detector using photodiode as photo sensitive element. Both technologies guarantee small form factor and lightweight detectors. The cost of both devices is acceptable. Our current design employs a PIN photodiode.

The diagram of the sensor used in our UAV is shown in Figure 9. This detector is sensitive to gamma and beta particles, also to accelerated protons and heavy ions and fast neutrons (Semkova et al., 2018). The electromagnetic and light shield of the sensor is made of $50 \mu \mathrm{m}$ copper foil supported by two printed circuit boards (PCBs). The photodiode and the internal electronics are soldered to the PCBs. The whole device is sprung suspended on the airframe of the UAV eliminating the detrimental effect of vibrations on its performance (see Figure 10). The sensor is developed around two compartments - one for the photodiode and one for the rest of the electronics.

The photodiode compartment is sealed (see Figure 9) - neither light nor external air is allowed to enter inside. The pressure changes are accommodated by the foil elasticity and pose no problem to the operation of the device. The chosen PIN photodiode is Hamamatsu S5107. It has $100 \mathrm{~mm}^{2}$ active area and $0.3 \mathrm{~mm}$ thickness of the sensitive silicon volume. The diode has maximum reverse voltage of $30 \mathrm{~V}$ and is reversely biased by $28 \mathrm{~V}$ power supply. The shielding of $50 \mu \mathrm{m}$ copper foil allows gamma rays with $60 \mathrm{keV}$ energy and higher to penetrate the device and be registered. Most beta particles are also readily captured. More details on the ionizing radiation sensor performance are disclosed in the next section.

The second compartment houses two amplifiers: a transimpedance amplifier that acts as a preamplifier of the weak signal coming directly from the photodiode; and an amplifier and signal shaper. The latter feeds the signal to one of the microcontroller's ADCs. The central frequency of the amplifier filter is $16 \mathrm{kHz}$.

We have configured the ADC to work in 12-bit mode with the maximum sampling frequency available $-530 \mathrm{kHz}$. Thus, we achieve a detailed image of each impulse. This makes the digital signal processing that follows efficient and accurate.

\section{Experimental Test}

We performed laboratory test in order to estimate the sensitivity of the sensor and its capabilities for spectral analysis. We conducted several tests using 5 different radioactive sources. Table 1 summarizes the radioactive sources used along with the counts per minute our device has registered when positioned in close proximity to each source. 
Table 1

Tested Radioactive Materials

\begin{tabular}{|c|c|c|}
\hline $\begin{array}{c}\text { Radioactive } \\
\text { source }\end{array}$ & Emits $\gamma$ and/or $\beta$ particles & $\begin{array}{c}\text { Counts } \\
\text { per minute }\end{array}$ \\
\hline Background & $\gamma+\beta$ & 5 \\
\hline Americium-241 & $\gamma$ & 590 \\
\hline Radium-226 & $\gamma+\beta$ & 1239 \\
\hline Uranium-238 & $\gamma+\beta$ & 48 \\
\hline Thorium-232 & $\gamma+\beta$ & 54 \\
\hline Potassium-40 & $\gamma+\beta$ & 15 \\
\hline
\end{tabular}

Figure 11 presents the spectra of the background radiation and the 5 radioactive sources we examined. There are 512 channels in the spectrum. All sources have wide spectra except Americium-241. The latter could be used to calibrate the sensor for $60 \mathrm{keV}$ gamma photons peaking in channel 1. All other channels are spaced proportionally to channel 1. Characteristic spectra are observed and it is clear that the device is usable for radioactive sources identification. It should be noted that besides Potassium-40 and Americium-241 all other sources contain radioactive daughter nuclides, most of which have reached dynamic equilibrium in the samples - for example the daughter nuclide Bismuth-214 in the Radium-226 and Uranium-238 samples. Observed characteristic behaviour of the PIN photodiode sensor is its low sensitivity to high energy gamma rays. 
Figure 11

Spectra of Different Radiation Sources Measured with the Sensor

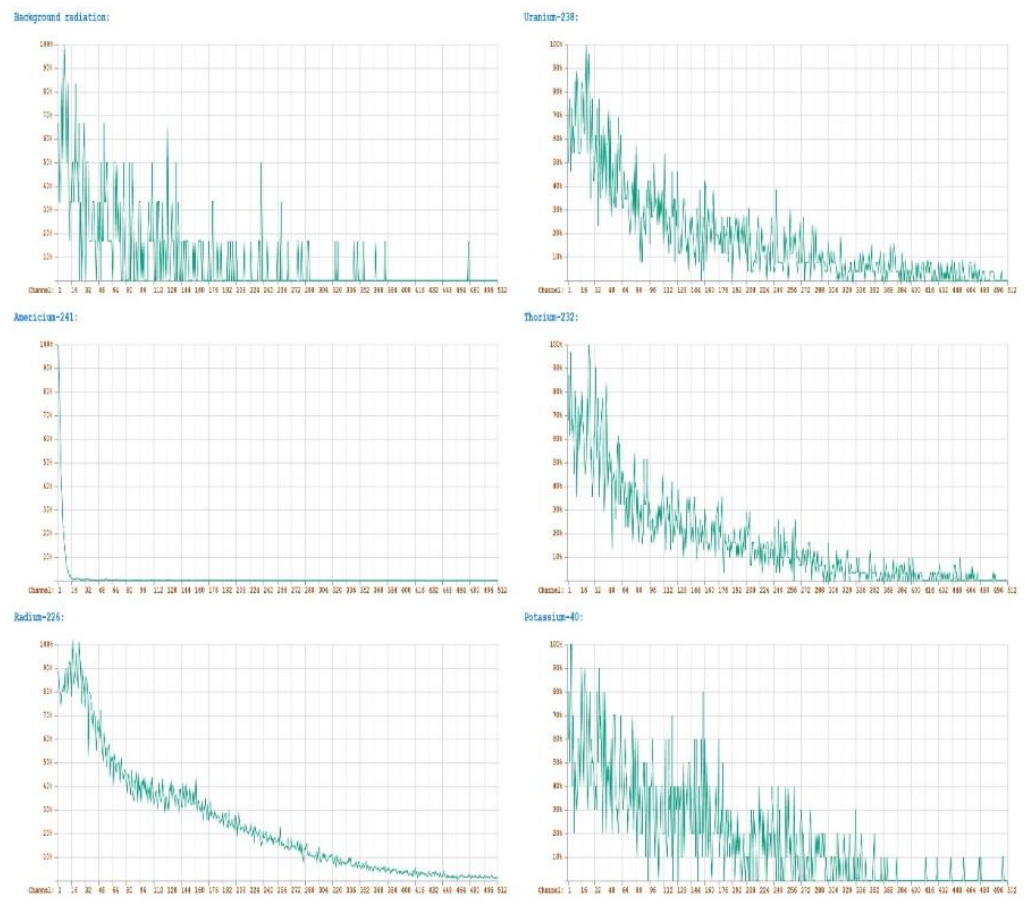

Conclusions and Future Work

Our successful results have encouraged us to continue work on unmanned systems for radiological surveying and on ionizing radiation sensors suitable for payloads. We plan on future development of the sensor and the UAV. Our 36-rotor aircraft requires means of navigating and route planning within buildings. The use of time-of-flight camera is being under consideration.

Another field of improvement is the radio communication within concrete buildings. We are considering supplementary radio links working on different frequencies that would yield longer range in such circumstances.

The radiation resistance of the UAV's electronics can be improved. The current microcontroller model installed in the autopilot may be replaced with a radiation resistant microcontroller. Employing radiation hardened device is not an option due to the prohibitively high cost of such parts. A possible substitute is an NXP Kinetis microcontroller having higher noise immunity. Worth considering is the MKE02Z64VQH4 model. It has been observed to work properly after receiving an X-ray dose of $30 \mathrm{krad}(\mathrm{Si})$. The microcontroller was subjected to $20 \mathrm{keV} \mathrm{X}$-rays (Leite et al., 2017). Although having lower processing power and no floating-point 
unit, it still offers a 32 bit ARM architecture and plenty of capabilities to perform the required tasks.

The ionizing radiation detector would be considerably improved when upgraded by adding a scintillation sensor to it. Both, the PIN photodiode and the scintillator would work in tandem. This approach is helpful in avoiding saturation of the scintillator. Such a saturation could occur under very high doses of radiation rendering the whole scintillator sensor useless. Functioning of the device under such circumstances could continue properly when the scintillator is supplemented by the current PIN photodiode-based sensor. The latter would continue to work as its saturation threshold is much higher than that of the scintillator device. The combination of PIN photodiode and scintillator increases the dynamic range of the apparatus. This way essentially the whole system is enabled to stay operational in large variety of scenarios. The scintillator is beneficial when investigating weak radioactive sources and searching for such at a distance. Another benefit of implementing a scintillation sensor is the increased sensitivity to high energy gamma rays, (a noticed drawback of the PIN photodiode-based device), and the ability to separate gamma rays from neutrons.

The ionizing radiation sensor is applicable to ground based robotic platforms as well. 


\section{References}

Anderson, C. (1990). Soviet official admits that robots couldn't handle Chernobyl cleanup. The Scientist. https://www.the-scientist.com/magazine/issue/ january-1990-4-2

Ashida, Y., Nagata, H., Koshio, Y., Nakaya, T., \& Wendell, R. (2018). Separation of gamma-ray and neutron events with $\mathrm{CsI}(\mathrm{Tl})$ pulse shape analysis. Progress of Theoretical and Experimental Physics, 043H01, 1-9. https://doi.org/10.1093/ptep/pty040

Battsengel, G., Geetha S., \& Jeon J. (2020). Analysis of technological trends and technological portfolio of unmanned aerial vehicle. Journal of Open Innovation: Technology, Market, and Complexity, 6(3), 1-14.

Berger, E. M. (2010). The Chernobyl disaster, concern about the environment, and life satisfaction. KYKLOS, 63(1), 1-8.

Bird, B., Griffiths, A., Martin, H., Codres, E., Jones, J., Stancu, A., Lennox, B., Watson, S., \& Poteau, X. (2019). A robot to monitor nuclear facilities. IEEE Robotics \& Automation Magazine, 1070-9932/19, 35-43.

Boudergui, K., Carrel, F., Domenech, T., Guénard, N., Poli, J.-P., Ravet, A., Schoepff, V., \& Woo, R. (2011). Development of a drone equipped with optimized sensors for nuclear and radiological risk characterization. 2011 2nd International Conference on Advancements in Nuclear Instrumentation, Measurement Methods and their Applications, Ghent, Belgium, 2011, 1-9.

Chaiyasoonthom, S., Hongyim, N., \& Mitatha, S. (2015). Building automatic packet report system to report position and radiation data for autonomous robot in the disaster area. 15th International Conference on Control, Automation and Systems (ICCAS 2015), Oct. 13-16, 2015, in BEXCO, Busan, Korea, 85-88.

Connor, D., Martin, P. G., \& Scott, T. B. (2016). Airborne radiation mapping: Overview and application of current and future aerial systems. International Journal of Remote Sensing, 37(24), 5953-5987.

Connor, D. T., Wood, K., Martin, P. G., Goren, S., Megson-Smith, D., Verbelen, Y., Chyzhevskyi, I., Kirieiev, S., Smith, N. T., Richardson, T., \& Scott, T. B. (2020). Radiological mapping of post-disaster nuclear environments using fixed-wing unmanned aerial systems: A study from Chornobyl. Frontiers in Robotics and AI, 6, 1-35.

Croon, G. de, \& Wagter, C. de. (2018). Challenges of autonomous flight in indoor environments. 2018 IEEE/RSJ International Conference on Intelligent Robots and Systems (IROS), Madrid, Spain, 1003-1009.

Dudar, A. M., Wagner, D. G., \& Teese, G. D. (1994). Autonomous mobile robot for radiological surveys. United States Patent, Patent Number: 5,324,948, Date of Patent: June 28, 1994. 
Garcia-Sanchez, A.-J., Angosto, E. A. G., Riquelme, P. A. M., Berna, A. S., \& Ramos-Amores, D. (2018). Ionizing radiation measurement solution in a hospital environment. Sensors, 18(510), 1-32.

Kawatsuma, S., Fukushima, M., \& Okada, T. (2012). Emergency response by robots to Fukushima-Daiichi accident: Summary and lessons learned. Industrial Robot, 39(5), 428-435.

Kawatsuma, S., Mimura, R., \& Asama, H. (2017). Unitization for portability of emergency response surveillance robot system: Experiences and lessons learned from the deployment of the JAEA-3 emergency response robot at the Fukushima Daiichi nuclear power plants. ROBOMECH J., 4(1), 1-7. doi:10.1186/s40648-017-0073-7

Khosiawan, Y., \& Nielsen, I. (2016). A system of UAV application in indoor environment. Production \& Manufacturing Research, 4(1), 2-22.

Kim, D., Woo, H., Ji, Y., Tamura, Y., Yamashita, A, \& Asama, H. (2017). 3D radiation imaging using mobile robot equipped with radiation detector. Proceedings of the 2017 IEEE/SICE International Symposium on System Integration, Taipei, Taiwan, December 11-14, 2017, 444-449.

Kovalev, I. V., Voroshilova, A. A., \& Karaseva, M. V. (2019). Analysis of the current situation and development trend of the international cargo UAVs market. Journal of Physics: Conference Series, 1399, 055095, 1-8.

Leite, F. G. H., Santos, R. B. B., Medina, N. H., \& Aguiar, V. A. (2017). Ionizing radiation effects on a COTS lowcost RISC microcontroller. 2017 18th IEEE Latin American Test Symposium (LATS), 1-4. doi:10.1109/LATW.2017.7906762

Marichal, G. N., Rodriguez, M. T., Hernández, Á., Rivera, S. C., \& Campoy, P. (2014). Vibration reduction for vision systems on board unmanned aerial vehicles using a neuro-fuzzy controller. Journal of Vibration and Control, 20(15), 2243-2253.

Martin P. G., Kwong, S., Smith, N. T., Yamashiki, Y., Payton, O. D., RussellPavier, F. S., Fardoulis, J. S., Richards, D. A., \& Scott, T. B. (2016). 3D unmanned aerial vehicle radiation mapping for assessing contaminant distribution and mobility. International Journal of Applied Earth Observation and Geoinformation, 52, 12-19.

Mochizuki, S., Kataoka, J., Tagawa, L., Iwamoto, Y., Okochi, H., Katsumi, N., Kinno, S., Arimoto, M., Maruhashi, T., Fujieda, K., Kuriharaa, T., \& Ohsukab, S. (2017). First demonstration of aerial gamma-ray imaging using drone for prompt radiation survey in Fukushima. Journal of Instrumentation, 12, 11014.

Molina, M., Campos, V., Montagud, M., \& Molina B. (2018). Ethics for civil indoor drones: A qualitative analysis. International Journal of Micro Air Vehicles, 10(4), 340-351. 
Nagatani, K., Kiribayashi, S., Okada, Y., Otake, K., Yoshida, K., Tadokoro, S., Nishimura, T., Yoshida, T., Koyanagi, E., Fukushima, M., \& Kawatsuma, S. (2013). Emergency response to the nuclear accident at the Fukushima Daiichi Nuclear Power Plants using mobile rescue robots. Journal of Field Robotics, 30(1), 44-63.

Nex, F., \& Remondino, F. (2019). Preface: Latest developments, methodologies, and applications based on UAV platforms. Drones, 3(1), 26, 1-3.

Piercy, J. E., \& Embleton, T. F. W. (1977). Review of noise propagation in the atmosphere. The Journal of the Acoustical Society of America, 61(6), 14031418.

Radkowski, S., \& Szulim, P. (2013). Analysis of vibration of rotors in unmanned aircraft. 2014 19th International Conference on Methods and Models in Automation and Robotics (MMAR), 748-753.

Seco, J., Clasie, B., \& Partridge, M. (2009). Review on the characteristics of radiation detectors for dosimetry and imaging. Physics in Medicine and Biology, 59(20), R303-R347.

Semkova, J., et al. (2018). Charged particles radiation measurements with LiulinMO dosimeter of FREND instrument aboard ExoMars trace gas orbiter during the transit and in high elliptic Mars orbit. Icarus, 303, 53-66.

Shrivastava, S., \& Henry, R. (2009). Process simulation and analysis for PIN detector. IEEE Circuits and Systems International Conference on Testing and Diagnosis, ICTD, 28-29 April 2009, 1-4.

Spur, F., \& Dachev, T. S. (2003). Long-term monitoring of the onboard aircraft exposure level with a si-diode based spectrometer. Advances in Space Research, 32(1), 53-58.

Spurný, F. (2005). Response of a si-diode-based device to fast neutrons. Radiation Measurements, 39, 219-223.

Spurný, F., Ploc, O., \& Jadrnickova, I. (2009). Spectrometry of linear energy transfer and dosimetry measurements onboard spacecrafts and aircrafts. Physics of Particles and Nuclei Letters, 6, 70-77.

Steinhaus, H. (1999). Mathematical snapshots (3rd ed). Dover.

Theys, B., Dimitriadis, G., Hendrick, P., \& DeSchutter, J. (2016). Influence of propeller configuration on propulsion system efficiency of multi-rotor Unmanned Aerial Vehicles. 2016 International Conference on Unmanned Aircraft Systems (ICUAS), 195-201.

Verbeke, J., \& Debruyne, S. (2016). Vibration analysis of a UAV multirotor frame. International Conference on Noise and Vibration Engineering, 23292338.

Yoon, S., Nasa, Diaz, P., Boyd, D., Chan, W., \& Theodore, C. R. (2017). Computational aerodynamic modeling of small quadcopter vehicles. 73rd 
Annual AHS International Forum and Technology Display, 2017 (AHS Forum 73), 1-16.

Zabunov, S. (2016). Quaternion-based autopilot for dodecacopters - Part I. Aerospace Research in Bulgaria, 28, 93-112.

Zabunov, S. (2019). Quaternion-based autopilot for dodecacopters - Part II. Aerospace Research in Bulgaria, 31, 91-98.

Zabunov, S., \& Mardirossian, G. (2018). Innovative dodecacopter design Bulgarian knight. International Journal of Aviation, Aeronautics, and Aerospace, 5(4), 1-16.

Zakaria, A. H., Mustafah, Y. M., Abdullah, J., Khair, N., \& Abdullah, T. (2016). Development of autonomous radiation mapping robot. 2016 IEEE International Symposium on Robotics and Intelligent Sensors, IRIS 2016, 17 20 December 2016, Tokyo, Japan, 81-86.

Zarowny, A. (2011). Lunokhod: From the moon to Chernobyl, the little robot that could!" Right Pundits, 2011. 